\title{
Relationship between Controlled Attenuation Parameter and Hepatic Steatosis as Assessed by Ultrasound in Alcoholic or Nonalcoholic Fatty Liver Disease
}

Jem Ma Ahn, Yong-Han Paik, Sin Yeong Min, Ju Yeon Cho, Won Sohn, Dong Hyun Sinn, Geum-Youn Gwak, Moon Seok Choi, Joon Hyeok Lee, Kwang Cheol Koh, Seung Woon Paik, and Byung Chul Yoo

Division of Gastroenterology and Hepatology, Department of Medicine, Samsung Medical Center, Sungkyunkwan University School of Medicine, Seoul, Korea

Background/Aims: The aim of this study was to evaluate the relationship between controlled attenuation parameter (CAP) and hepatic steatosis, as assessed by ultrasound (US) in patients with alcoholic liver disease (ALD) or non-alcoholic fatty liver disease (NAFLD). Methods: Patients with either ALD or NAFLD who were diagnosed with fatty liver with US and whose CAP scores were measured, were retrospectively enrolled in this study. The degree of hepatic steatosis assessed by US was categorized into mild (S1), moderate (S2), and severe (S3). Results: A total of 186 patients were included: 106 with NAFLD and 80 with ALD. Regarding hepatic steatosis, the CAP score was significantly correlated with US ( $\rho=0.580, p<0.001)$, and there was no significant difference between the NAFLD and ALD groups ( $\rho=0.569$, $p<0.001 ; \rho=0.519, p<0.001 ; p=0.635)$. Using CAP, area under receiver operating characteristic curves for $\geq \mathrm{S} 2$ and $\geq \mathrm{S} 3$ steatosis were excellent $(0.789$ and 0.843 , respectively). For sensitivity $\geq 90 \%$, CAP cutoffs for the detection of $\geq S 2$ and $\geq \mathrm{S} 3$ steastosis were separated with a gap of approximately $35 \mathrm{~dB} / \mathrm{m}$ in all patients and in each of the NAFLD and ALD groups. Conclusions: The CAP score is well correlated with hepatic steatosis, as assessed by US, in both ALD and NAFLD. (Gut Liver 2016;10:295-302)

Key Words: Steatosis; Ultrasonography; Controlled attenuation parameter; Liver diseases, alcoholic; Non-alcoholic fatty liver disease

\section{INTRODUCTION}

Hepatic steatosis is one of the most common conditions in chronic liver disease, with an increasing prevalence of $16 \%$ to
45\% in Western society, $9 \%$ to $29 \%$ in Eastern society, $76 \%$ in the obese and $46 \%$ to $80 \%$ in heavy alcoholics. ${ }^{1-3}$ Alcoholic liver disease (ALD), induced by excessive alcohol consumption, and nonalcoholic fatty liver disease (NAFLD), caused by obesity and insulin resistance, are the most common diseases associated with hepatic steatosis, although hepatic steatosis is also found in patients with hepatitis $\mathrm{C}$ virus infection and those receiving certain medications, such as corticosteroids and amiodarone. ${ }^{4,5}$ Regardless of etiology, hepatic steatosis can progress to end stage liver disease such as liver cirrhosis or hepatocellular carcinoma. ${ }^{1,6-8}$ Moreover, NAFLD has emerged as one of the risk factors for coronary artery disease and type 2 diabetes mellitus. ${ }^{9}$ Patients with NAFLD also show higher mortality from both liver related and non-liver-related death compared to the general population ${ }^{1}$. Thus, early diagnosis and proper management of hepatic steatosis are important.

Until now, liver biopsy is regarded as the gold standard for assessing hepatic steatosis. ${ }^{1,2,7}$ However, biopsy has several limitations such as invasiveness, intraobserver/interobserver variability, severe procedure-related complications, and sampling error. It is also difficult to repeat during follow-up due to its limitations. ${ }^{7,10,11}$ Thus, hepatic steatosis is mainly diagnosed by noninvasive means, using imaging modalities including ultrasound (US), computerized tomography, magnetic resonance imaging, and magnetic resonance spectroscopy in real practice. ${ }^{12,13}$ Among these, US is the most commonly used imaging tool to detect hepatic steatosis. In a previous study, US has 92\% sensitivity and 100\% specificity for the detection of hepatic steatosis compared to the use of biopsy as the standard method. ${ }^{14}$ Another study also demonstrated that US combined with ultrasonographic images has both high sensitivity and specificity for evaluating hepatic steatosis when fat content is over $20 \%{ }^{15}$

Correspondence to: Yong-Han Paik

Department of Medicine, Samsung Medical Center, Sungkyunkwan University School of Medicine, 81 Irwon-ro, Gangnam-gu, Seoul 135-710, Korea

Tel: +82-2-3410-3878, Fax: +82-2-3410-6983, E-mail: yh.paik@skku.edu

Received on March 30, 2015. Revised on May 20, 2015. Accepted on May 20, 2015. Published online September 9, 2015

pISSN 1976-2283 eISSN 2005-1212 http://dx.doi.org/10.5009/gnl15155

(a) This is an Open Access article distributed under the terms of the Creative Commons Attribution Non-Commercial License (http://creativecommons.org/licenses/by-nc/4.0) which permits unrestricted non-commercial use, distribution, and reproduction in any medium, provided the original work is properly cited. 
Transient elastography was recently introduced as a promising noninvasive quantitative method for measuring steatosis. In transient elastography, hepatic steatosis is determined by the controlled attenuation parameter (CAP) and hepatic fibrosis is determined by liver stiffness measurement (LSM). ${ }^{16-19}$ This modality is objective and operator-/machine- independent. CAP can be used to assess hepatic steatosis from 10\% of fat content. Moreover CAP is remarkably correlated with histological steatosis grade as well as \% of lipid droplets in hepatocytes. ${ }^{5,16,17,20-22} \mathrm{~A}$ recent study reported that CAP is also well correlated with hepatic steatosis assessed by US in the general population $(\rho=0.73$, $\mathrm{p}<0.001){ }^{23}$ However, there has been no study investigating the relationship between CAP and hepatic steatosis assessed by US regarding each etiology of hepatic steatosis. Thus, the aim of this study was to evaluate the relationship of CAP to hepatic steatosis assessed by US in patients with either NAFLD or ALD.

\section{MATERIAL AND METHODS}

\section{Patients}

From August 2012 to January 2014, 2,964 patients received transient elastography at Samsung Medical Center, Seoul, Korea. Among them, 244 patients, who were diagnosed with fatty liver by US with etiology of either NAFLD or ALD and subsequently received transient elastography within 1 month, were enrolled in this study. ALD was diagnosed when patients had moderate to severe alcohol consumption history ( $>40 \mathrm{~g} /$ day for women and $>60 \mathrm{~g} /$ day for men) concurrent with fatty liver on US. ${ }^{24}$ On the other hand, NAFLD was diagnosed when patients showing fatty liver in US had less than minimal alcohol consumption history (<20-30 g/day) and no other well-known causes of fatty liver such as viral hepatitis or certain medications. ${ }^{24}$ A total of 58 patients were excluded with the following criteria: (1) presence of other etiologies of liver disease such as viral, toxic, genetic, and autoimmune hepatitis; (2) presence of hepatic malignancy; (3) unreliable or failed LSM or CAP, (4) overlapping etiologies of hepatic steatosis; and (5) lack of data. After exclusion, a total of 186 patients were analyzed in this study.

\section{Ultrasound}

Abdominal US was performed to assess hepatic steatosis. Hepatic steatosis was categorized into three groups of mild (S1), moderate (S2), and severe (S3) hepatic steatosis based on the following US features: increased echogenicity in liver parenchyma, altered hepatorenal contrast, deep attenuation, and blurred blood vessel. ${ }^{14,15}$

\section{LSM and CAP}

Both LSM and CAP were assessed using the transient elastography by one professionally trained technician. The evaluation was performed on the right lobe of the liver through intercostal spaces on patients lying in the decubitus position with the right arm in abduction. Median LSM was expressed in kilopascals $(\mathrm{kPa})$. We considered LSM reliable only when LSM was acquired from at least 10 valid measurements, with a success rate of over $60 \%$, and with interquartile range/median (IQR/M) less than 0.3. Regarding CAP, ultrasonic attenuation was measured at 3.5 $\mathrm{MHz}$ using signals assessed by transient elastography. Median CAP was expressed in decibel per meter $(\mathrm{dB} / \mathrm{m})$. The CAP was only calculated when LSM was simultaneously valid for the same signals. Also we considered CAP reliable with both IQR/M less than 0.3 and success rate greater than 60\%.

\section{Assessment of lifestyle and biochemical parameters}

At the time of transient elastography, we collected patient information on life-style (exercise [duration, frequency, type], alcohol consumption [quantity, frequency, type], and smoking [none, past, current, pack-years]) through questionnaires. The body mass index (BMI) was calculated as body weight in kilograms divided by height in meters squared $\left(\mathrm{kg} / \mathrm{m}^{2}\right)$. Laboratory data including liver biochemistry (aspartate aminotransferase [AST], alanine aminotransferase [ALT], alkaline phosphatase [ALP], $\gamma$-glutamyltransferase [GGT], total bilirubin [TB], albumin [Alb]), platelet count (PLT), and total cholesterol (TC) were performed within 1 month from the date of transient elastography.

\section{Statistical analysis}

Continuous variables are expressed as means \pm standard deviation or median (interquartile range) whereas categorical variables are expressed as number (\%). Univariate linear regression analysis were performed to identify the factors influencing CAP (age, gender, BMI, exercise, smoking, LSM, steatosis grade in US, PLT, AST, ALT, ALP, Alb, GGT, TB, TC), followed by multivariate analysis using the factors with $\mathrm{p}<0.2$ in univariate linear regression analysis. Spearman correlation coefficient was used to investigate the correlation between CAP and steatosis grade by US while z-test was utilized for evaluating the difference in correlation between the NAFLD and ALD groups. The performance of CAP for detecting each steatosis grade in US was assessed using the area under receiver operating characteristic curves (AUROC). We calculated the sensitivity (Se), specificity (Sp), positive predictive value (PPV), and negative predictive value (NPV) at the cutoff values of CAP maximizing Sp in situations with $\mathrm{Se}>90 \%$ for each steatosis grade. A p-value less than 0.05 was considered significant. All analyses were performed using SPSS software version 21.0 for Windows (SPSS Inc., Chicago, IL, USA).

\section{Ethics statement}

The study was approved by the Institutional Review Board of Samsung Medical Center, Seoul, Korea (IRB number: 2014-07006-002). Informed consent was waived by the IRB. 


\section{RESULTS}

\section{Patient characteristics}

Table 1 shows the baseline characteristics of the study population. In a total of 186 patients with fatty liver, 106 had NAFLD and 80 had ALD. The mean age of all patients was $53.4 \pm 12.0$ years and males were dominant (76.9\%). The mean LSM was $7.0 \pm 4.0 \mathrm{kPa}$, while the mean and median CAP were $281.6 \pm 46.0$ and 279.5 (251.0 to 313.3 ) dB/m, respectively. Regarding the steatosis grades in US, 75 patients (40.3\%) had S1 steatosis, 76 (40.9\%) had S2, and 35 (18.8\%) had S3.

In subgroup analysis according to etiology, males were more dominant in the ALD group than in the NAFLD group (95.0\% vs $63 \%, p<0.001)$. The NAFLD group had higher mean and median CAP than the ALD group $(288.8 \pm 46.0 \mathrm{~dB} / \mathrm{m}$ vs $272.0 \pm 44.5$ $\mathrm{dB} / \mathrm{m}, \mathrm{p}=0.014 ; 293.0$ [256.8 to 322.3] dB/m vs 269.5 [244.5 to 299.0$] \mathrm{dB} / \mathrm{m}, \mathrm{p}=0.008$ ), but there was no difference in mean and median LSM between the NAFLD and ALD groups $(7.0 \pm 4.3$
$\mathrm{kPa}$ vs $7.0 \pm 3.5 \mathrm{kPa}, \mathrm{p}=0.611 ; 5.9 \mathrm{kPa}$ [4.8 to 7.7 ] vs 5.9 [4.9 to $8.6] \mathrm{kPa}, \mathrm{p}=0.833)$. The NAFLD group also had a higher proportion of moderate to severe steatosis in US than the ALD group $(\mathrm{p}<0.001)$ : in the NAFLD group, 30 patients $(28.3 \%)$ had S1 steatosis, 49 (46.2\%) had S2, and 27 (25.5\%) had S3; in the ALD group, 45 (56.2\%) had S1, 27 (33.8\%) had S2, and eight (10.0\%) had S3. In the laboratory data, only mean GGT differed between the NAFLD and ALD groups $(50.3 \pm 45.1 \mathrm{U} / \mathrm{L}$ vs $95.7 \pm 91.4 \mathrm{U} / \mathrm{L}$, $\mathrm{p}<0.001)$.

\section{Factors associated with CAP}

In univariate linear regression analysis, $A l b(\beta=36.0$, $p=0.008)$, steatosis grade in US $(\beta=35.8, p<0.001)$, BMI $(\beta=5.7$, $p<0.001)$, LSM $(\beta=2.0, p=0.019)$, ALT $(\beta=0.4, p<0.001)$, age $(\beta=-$ $0.9, p=0.001)$, and regular exercise $(\beta=-17.0, p=0.012)$ were associated with CAP in all patients (Table 2). Among these factors, steatosis grades in US and BMI were independent factors related with CAP in multivariate linear regression analysis $(\beta=28.9$,

Table 1. Baseline Characteristics of Patients

\begin{tabular}{|c|c|c|c|c|}
\hline Characteristic & All patients $(n=186)$ & NAFLD $(n=106)$ & $\operatorname{ALD}(\mathrm{n}=80)$ & p-value \\
\hline Age, yr & $53.4 \pm 12.0$ & $48.8 \pm 13.3$ & $52.5 \pm 9.8$ & 0.092 \\
\hline Male sex & 143 (76.9) & $67(63.2)$ & 76 (95.0) & $<0.001$ \\
\hline BMI, $\mathrm{kg} / \mathrm{m}^{2}$ & $26.4 \pm 3.5$ & $26.8 \pm 3.7$ & $25.8 \pm 3.3$ & 0.102 \\
\hline Current smoker & 47 (25.3) & $16(15.1)$ & $31(38.8)$ & $<0.001$ \\
\hline Regular exercise & $99(53.2)$ & $56(52.8)$ & $43(53.8)$ & 0.901 \\
\hline \multicolumn{5}{|l|}{ LSM, kPa } \\
\hline Mean & $7.0 \pm 4.0$ & $7.0 \pm 4.3$ & $7.0 \pm 3.5$ & 0.611 \\
\hline Median (IQR) & $5.9(4.9-7.8)$ & $5.9(4.8-7.7)$ & $5.9(4.9-8.6)$ & 0.833 \\
\hline \multicolumn{5}{|l|}{$\mathrm{CAP}, \mathrm{dB} / \mathrm{m}$} \\
\hline Mean & $281.6 \pm 46.0$ & $288.8 \pm 46.0$ & $272.0 \pm 44.5$ & 0.014 \\
\hline Median (IQR) & $279.5(251.0-313.3)$ & $293.0(256.8-322.3)$ & 269.5 (244.5-299.0) & 0.008 \\
\hline Steatosis grade in US & & & & $<0.001$ \\
\hline $\mathrm{S} 1$ & $75(40.3)$ & $30(28.3)$ & $45(56.2)$ & \\
\hline $\mathrm{S} 2$ & 76 (40.9) & $49(46.2)$ & $27(33.8)$ & \\
\hline S3 & 35 (18.8) & $27(25.5)$ & $8(10.0)$ & \\
\hline PLT, $10^{9} / \mathrm{L}$ & $210.8 \pm 57.1$ & $215.4 \pm 54.0$ & $204.7 \pm 60.7$ & 0.137 \\
\hline PT INR & $0.97 \pm 0.09$ & $0.96 \pm 0.05$ & $0.98 \pm 0.12$ & 0.884 \\
\hline $\mathrm{TC}, \mathrm{mg} / \mathrm{mL}$ & $184.9 \pm 34.8$ & $186.3 \pm 33.2$ & $183.1 \pm 36.9$ & 0.534 \\
\hline $\mathrm{TB}, \mathrm{mg} / \mathrm{dL}$ & $0.79 \pm 0.46$ & $0.80 \pm 0.57$ & $0.78 \pm 0.28$ & 0.083 \\
\hline AST, U/L & $35.4 \pm 19.4$ & $34.5 \pm 18.8$ & $36.7 \pm 20.2$ & 0.660 \\
\hline ALT, U/L & $45.4 \pm 31.4$ & $48.1 \pm 32.3$ & $41.9 \pm 30.2$ & 0.110 \\
\hline ALP, U/L & $65.8 \pm 18.8$ & $67.2 \pm 20.0$ & $64.0 \pm 17.0$ & 0.288 \\
\hline GGT, U/L & $69.8 \pm 72.3$ & $50.3 \pm 45.1$ & $95.7 \pm 91.4$ & $<0.001$ \\
\hline $\mathrm{Alb}, \mathrm{g} / \mathrm{dL}$ & $4.6 \pm 0.2$ & $4.6 \pm 0.2$ & $4.6 \pm 0.2$ & 0.330 \\
\hline
\end{tabular}

Data are presented as mean \pm SD or median (interquartile range).

NAFLD, nonalcoholic fatty liver disease; ALD, alcoholic liver disease; BMI, body mass index; LSM, liver stiffness measurement; IQR, interquartile range; CAP, controlled attenuation parameter; US, ultrasound; S1, mild steatosis; S2, moderate steatosis; S3, severe steatosis; PLT, platelet; PT INR, prothrombin time international normalized ratio; TC, total cholesterol; TB, total bilirubin; AST, aspartate aminotransferase; ALT, alanine aminotransferase; ALP, alkaline phosphatase; GGT, $\gamma$-glutamyl transpeptidase; Alb, albumin. 
Table 2. Factors Influencing Controlled Attenuation Parameter Values Using Univariate and Multivariate Linear Regression Analysis

\begin{tabular}{|c|c|c|c|c|c|c|c|c|c|c|c|c|}
\hline \multirow{3}{*}{ Variable } & \multicolumn{4}{|c|}{ All patients } & \multicolumn{4}{|c|}{ NAFLD } & \multicolumn{4}{|c|}{ ALD } \\
\hline & \multicolumn{2}{|c|}{ Uni } & \multicolumn{2}{|c|}{ Multi } & \multicolumn{2}{|c|}{ Uni } & \multicolumn{2}{|c|}{ Multi } & \multicolumn{2}{|c|}{ Uni } & \multicolumn{2}{|c|}{ Multi } \\
\hline & $\beta$ & $\mathrm{p}$ & $\beta$ & $\mathrm{p}$ & $\beta$ & $\mathrm{p}$ & $\beta$ & $\mathrm{p}$ & $\beta$ & $\mathrm{p}$ & $\beta$ & $\mathrm{p}$ \\
\hline Age & -0.9 & 0.001 & -0.07 & 0.788 & -0.9 & 0.006 & -0.2 & 0.429 & -0.6 & 0.284 & - & - \\
\hline Regular exercise & -17.0 & 0.012 & -4.1 & 0.461 & -22.0 & 0.013 & -1.5 & 0.847 & -10.0 & 0.322 & - & - \\
\hline BMI & 5.7 & $<0.001$ & 3.2 & $<0.001$ & 4.7 & $<0.001$ & 2.0 & 0.109 & 6.8 & $<0.001$ & 5.3 & $<0.001$ \\
\hline LSM & 2.0 & 0.019 & 0.7 & 0.297 & 1.8 & 0.091 & 0.8 & 0.390 & 2.4 & 0.091 & 2.3 & 0.068 \\
\hline Steatosis in US & 35.8 & $<0.001$ & 28.9 & $<0.001$ & 36.3 & $<0.001$ & 30.8 & $<0.001$ & 34.4 & $<0.001$ & 22.9 & 0.001 \\
\hline PLT & 0.1 & 0.083 & -0.001 & 0.991 & 0.2 & 0.020 & 0.01 & 0.941 & -0.02 & 0.832 & - & - \\
\hline PT INR & 31.4 & 0.403 & - & - & -44.7 & 0.595 & - & - & 63.0 & 0.123 & 246.2 & 0.173 \\
\hline ALT & 0.4 & $<0.001$ & 0.03 & 0.768 & 0.3 & 0.013 & -0.05 & 0.728 & 0.4 & 0.009 & -0.1 & 0.372 \\
\hline ALP & -0.2 & 0.313 & - & - & 0.07 & 0.775 & - & - & -0.8 & 0.010 & -0.2 & 0.463 \\
\hline GGT & -0.01 & 0.913 & - & - & 0.2 & 0.062 & 0.1 & 0.185 & -0.02 & 0.782 & - & - \\
\hline Alb & 36.0 & 0.008 & 12.3 & 0.286 & 20.7 & 0.255 & - & - & 51.8 & 0.011 & 18.6 & 0.335 \\
\hline $\mathrm{TC}$ & 0.09 & 0.365 & - & - & 0.05 & 0.320 & - & - & -0.2 & 0.190 & -0.1 & 0.361 \\
\hline
\end{tabular}

NAFLD, nonalcoholic fatty liver disease; ALD, alcoholic liver disease; uni, univariate linear regression analysis; multi, multivariate linear regression analysis; $\beta$, beta coefficient; BMI, body mass index; LSM, liver stiffness measurement; US, ultrasound; PLT, platelet; PT INR, prothrombin time international normalized ratio; ALT, alanine aminotransferase; ALP, alkaline phosphatase; GGT, $\gamma$-glutamyl transpeptidase; Alb, albumin; TC, total cholesterol.

A

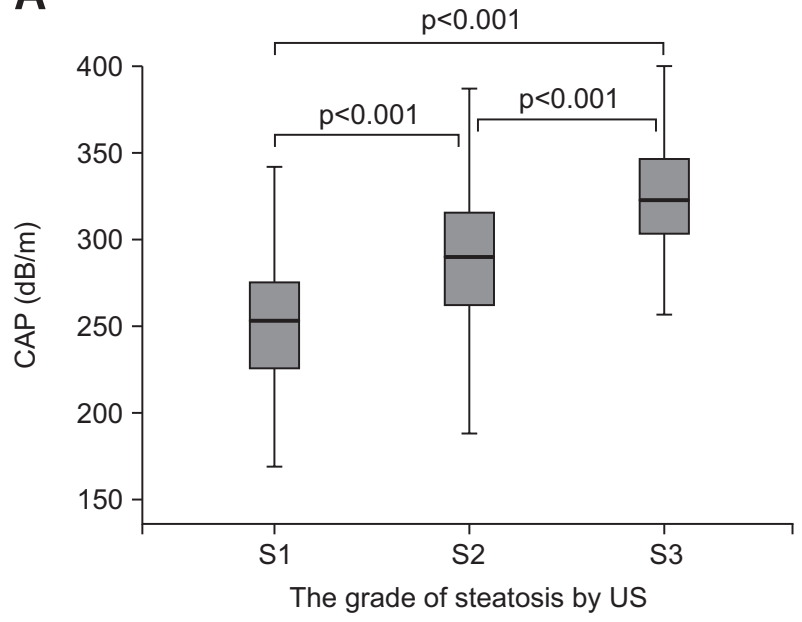

C

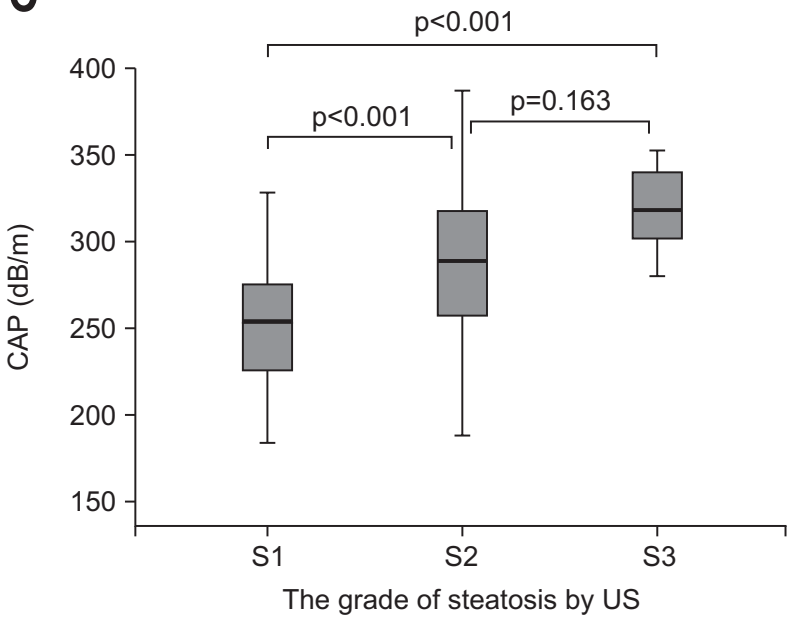

B

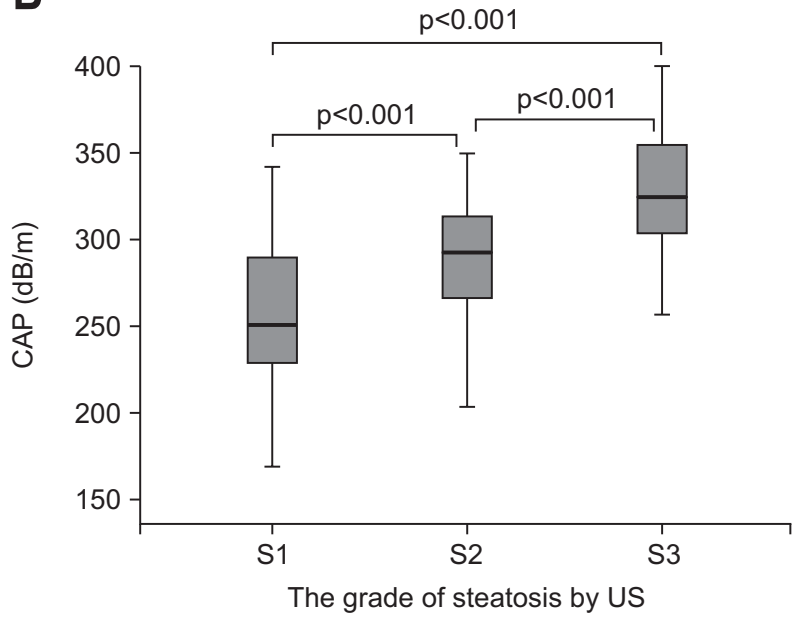

Fig. 1. The distribution of controlled attenuation parameter (CAP) according to steatosis grades as assessed by ultrasound (US) in all patients (A), patients with nonalcoholic fatty liver disease (B), and patients with alcoholic liver disease (C). 
$\mathrm{p}<0.001 ; \beta=3.2, \mathrm{p}<0.001$ ) (Table 2). CAP scores increased by $28.9 \mathrm{~dB} / \mathrm{m}$ with every steatosis grade increase in US, while CAP scores rose by $3.2 \mathrm{~dB} / \mathrm{m}$ with every $1 \mathrm{~kg} / \mathrm{m}^{2}$ increase in BMI.

In subgroup analysis according to etiology, CAP was related with steatosis grade in US $(\beta=36.3, p<0.001)$, BMI $(\beta=4.7$, $\mathrm{p}<0.001)$, ALT $(\beta=0.3, p=0.013)$, platelet $(\beta=0.2, p=0.020)$, age $(\beta=-0.9, p=0.006)$, and regular exercise $(\beta=-22.0, p=0.013)$ in patients with NAFLD; in patients with ALD, CAP was related with Alb $(\beta=51.8, p=0.011)$, steatosis grades in US $(\beta=34.4$, $\mathrm{p}<0.001)$, BMI $(\beta=6.8, p<0.001)$, ALT $(\beta=0.4, p=0.009)$, and ALP $(\beta=-0.8, p=0.010)$ (Table 2). Among these parameters, steatosis grades in US was the independent factor associated with CAP with high $\beta$ coefficient in both NAFLD and ALD groups using multivariate linear regression analysis $(\beta=30.8, p<0.001 ; \beta=22.9$, $\mathrm{p}=0.001$ ) (Table 2). Additionally, BMI was independently related with CAP only in patients with ALD but its $\beta$ coefficient was relatively low $(\beta=5.3, p<0.001)$ (Table 2$)$.

\section{Relationship between CAP and steatosis grade in US}

Median CAP for each steatosis grade in US had a positive dose response relationship (Fig. 1): in all patients (S1, 253.0 $\mathrm{dB} / \mathrm{m}$; S2, $290.0 \mathrm{~dB} / \mathrm{m}$; S3, $323.0 \mathrm{~dB} / \mathrm{m}$ ), in NAFLD subgroup (S1, $250.5 \mathrm{~dB} / \mathrm{m}$; S2, $292.0 \mathrm{~dB} / \mathrm{m}$; S3, $324.5 \mathrm{~dB} / \mathrm{m}$ ), and in ALD subgroup (S1, $254.0 \mathrm{~dB} / \mathrm{m}$; S2, $289.0 \mathrm{~dB} / \mathrm{m}$; S3, $317.5 \mathrm{~dB} / \mathrm{m}$ ). There was no significant difference in the median CAP of each steatosis grade between NAFLD and ALD groups ( $\mathrm{S} 1, \mathrm{p}=0.826$; $\mathrm{S} 2, \mathrm{p}=0.838$; S3, $\mathrm{p}=0.502$ ). Furthermore, CAP was significantly correlated with steatosis grade by US in both NAFLD and ALD groups $(\rho=0.569, p<0.001 ; \rho=0.519, p<0.001)$ as well as in all patients $(\rho=0.580, p<0.001)$, with no significant difference between etiologies $(\mathrm{p}=0.635)$.

\section{Diagnostic performance of CAP for assessing hepatic steatosis}

Fig. 2 represents ROC curves for assessing hepatic steatosis grades ( $\geq \mathrm{S} 2$ or $\geq \mathrm{S} 3$ steatosis) with CAP. Table 3 also shows the diagnostic performance of CAP for assessing hepatic steatosis. For detecting each grade of hepatic steatosis, AUROCs for both $\geq \mathrm{S} 2$ and $\geq \mathrm{S} 3$ steatosis were excellent (total patients: 0.789, 0.843; NAFLD group: 0.791, 0.823; ALD group: 0.780, 0.867). The optimal cutoff values for $\geq \mathrm{S} 2$ steatosis with a condition of Se $>90 \%$ were $250.0 \mathrm{~dB} / \mathrm{m}$ in all patients $(90.1 \%$ Se, $45.3 \%$ Sp, 70.9\% PPV, 75.6\% NPV), $255.5 \mathrm{~dB} / \mathrm{m}$ in the NAFLD group (90.8\% Se, 60.0\% Sp, 85.2\% PPV, 72.0\% NPV), and $245.0 \mathrm{~dB} /$ $\mathrm{m}$ in the ALD group (91.4\% Se, 62.2\% Sp, 53.3\% PPV, 85.0\% $\mathrm{NPV}$ ); for $\geq \mathrm{S} 3$ steatosis, $289.5 \mathrm{~dB} / \mathrm{m}$ in all patients $(91.4 \% \mathrm{Se}$, 66.2\% Sp, 38.6\% PPV, 97.1\% NPV), $290.5 \mathrm{~dB} / \mathrm{m}$ in the NAFLD group (92.6\% Se, 59.5\% Sp, 43.9\% PPV, 95.9\% NPV) and 279.5 $\mathrm{dB} / \mathrm{m}$ in the ALD group (100\% Se, 68.1\% Sp, 25.8\% PPV, 100\% NPV). The gap between the cutoff values of S2 and S3 steatosis was about $35 \mathrm{~dB} / \mathrm{m}$ in all three groups. Also the NAFLD group had approximately $10 \mathrm{~dB} / \mathrm{m}$ higher cut-off values consistently in each steatosis grade than the ALD group in same degree of BMI.

\section{DISCUSSION}

Although liver biopsy is considered the gold standard for assessing hepatic steatosis, biopsy is rarely performed due to its limitations. Consequently, steatosis is mainly evaluated using noninvasive techniques. Among these techniques, CAP is a recent, novel, and noninvasive method for both diagnosing and quantifying hepatic steatosis with accuracy, convenience, and economic feasibility, whereas US is the most commonly used

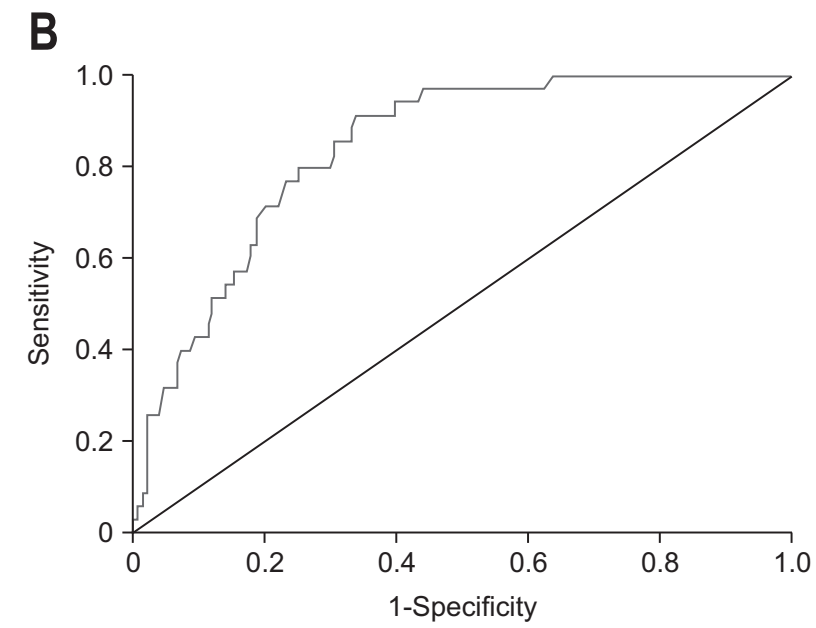

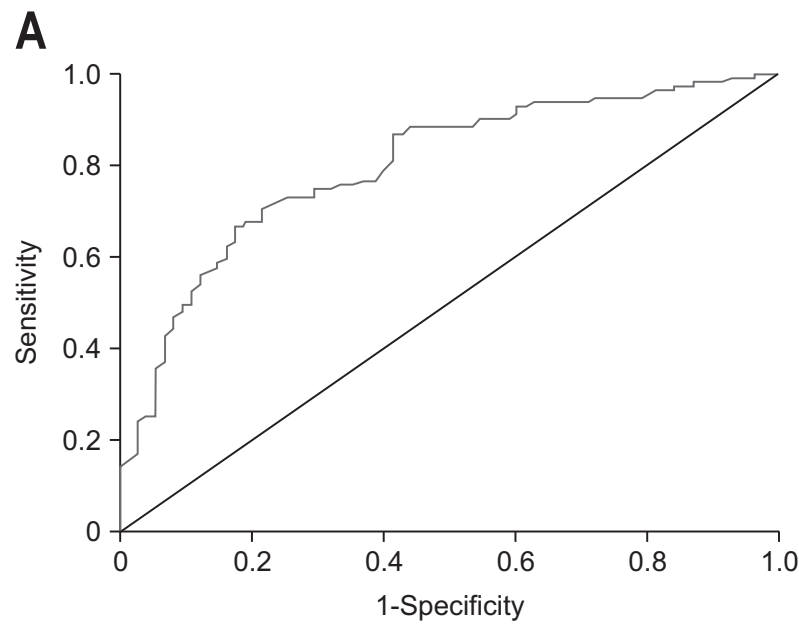

Fig. 2. Receiver operating characteristic curves and area under receiver operating characteristic curves (AUROCs) for the detection of $\geq \mathrm{S} 2$ and $\geq \mathrm{S} 3$ steatosis using controlled attenuation parameter (CAP). Using CAP, the AUROCs (95\% confidence interval) for $\geq \mathrm{S} 2$ and $\geq \mathrm{S} 3$ steatosis were (A) 0.80 (0.73 to 0.86 ) and (B) 0.84 (0.78 to 0.90 ) in all patients, respectively.

S2, moderate steatosis; S3, severe steatosis. 
Table 3. Diagnostic Performance of Controlled Attenuation Parameter for the Detection of $\geq \mathrm{S} 2$ and $\geq \mathrm{S} 3$ Steatosis

\begin{tabular}{lccc}
\hline & All patients & NAFLD & ALD \\
\hline S2 steatosis & & & $0.780(0.675-0.885)$ \\
AUROC $(95 \%$ CI) & $0.789(0.735-0.863)$ & $0.791(0.691-0.892)$ & 245.0 \\
CAP cutoff, dB/m & 250.0 & 255.5 & 91.4 \\
Sensitivity, \% & 90.1 & 90.8 & 62.2 \\
Specificity, \% & 45.3 & 60.0 & 53.3 \\
PPV, \% & 70.9 & 85.2 & 85.0 \\
NPV, \% & 75.6 & 72.0 & \\
$\geq$ S3 steatosis & & & $0.867(0.777-0.957)$ \\
AUR0C (95\% CI) & $0.843(0.782-0.904)$ & 0.723 \\
CAP cutoff, dB/m & 289.5 & 290.5 & 279.5 \\
Sensitivity, \% & 91.4 & 92.6 & 100.0 \\
Specificity, \% & 66.2 & 59.5 & 68.1 \\
PPV, \% & 38.6 & 43.9 & 25.8 \\
NPV, \% & 97.1 & 95.9 & 100.0 \\
\hline
\end{tabular}

NAFLD, nonalcoholic fatty liver disease; ALD, alcoholic liver disease; S2, moderate steatosis; AUROC, area under receiver operating characteristic curves; CI, confidence interval; CAP, controlled attenuation parameter; PPV, positive predictive value; NPV, negative predictive value; S3, severe steatosis.

method for assessing hepatic steatosis. Our study attempted to evaluate the relationship between CAP and the steatosis grade assessed by US in patients with NAFLD or ALD as well as to identify differences in this relationship between the NAFLD and ALD groups.

In our study, CAP is significantly related with the steatosis grade assessed by US regardless of the fatty liver etiology. Correlation coefficients between CAP and steatosis grade in US were greater than 0.5 and there was no significant difference between the fatty liver etiologies. These results were consistent with previous data suggesting significant correlation between CAP and steatosis grade assessed by US $(\rho=0.73, p<0.001) .{ }^{23}$ Of note, our study was the first to identify the relationship between CAP and steatosis grade in US according to fatty liver etiology. Our correlation coefficient was a little bit lower than that of previous studies. This discrepancy may be explained by differences in both study populations and classification criteria of the steatosis grades assessed by US. Median CAP also showed positive dose-response relationships in all patients and in both subgroups. Thus, with these results, we can conclude that CAP is considerably associated with steatosis grade assessed by US as well as histological steatosis severity, regardless of etiology.

With regard to CAP performance, our results showed excellent diagnostic accuracy. Both NAFLD and ALD groups had similarly high CAP diagnostic accuracy for $\geq \mathrm{S} 2$ steatosis (AUROC, 0.79 in NAFLD vs 0.78 in ALD) and $\geq \mathrm{S} 3$ steatosis (AUROC, 0.82 in NAFLD vs 0.87 in ALD). These findings were consistent with previous results showing no difference in CAP accuracy for assessing steatosis between etiologies including chronic hepatitis $\mathrm{B}$, chronic hepatitis $\mathrm{C}$, and NAFLD using biopsy as the standard method. ${ }^{22}$

In our study, the optimal cutoff values for detecting $\geq \mathrm{S} 2$ and $\geq \mathrm{S} 3$ steatosis were determined when the values indicated the highest Sp given that Se was greater than 90\%. Consequently, fewer than $10 \%$ of the patients would be excluded from the diagnosis of moderate or severe fatty liver. These cutoff values are separated for each grade of steatosis, with a gap of about $35 \mathrm{~dB} /$ $\mathrm{m}$ in all patients and each subgroup. In addition, $\beta$ coefficient between CAP and steatosis grades in multi linear regression analysis showed comparable values around 30 . These results were similar to those from a previous study with 112 patients in France, in which cutoff values were $252 \mathrm{~dB} / \mathrm{m}$ for $\geq \mathrm{S} 2$ steatosis and $296 \mathrm{~dB} / \mathrm{m}$ for $\geq \mathrm{S} 3$ steatosis. ${ }^{20}$ However our cutoff values differed from those in other studies. These differences may be because the study populations were different and the previous studies used the optimal cutoff values for maximizing the Youden index..$^{5,21,23}$ In our study, there was no marked difference in Se and Sp for both $\geq \mathrm{S} 2$ and $\geq \mathrm{S} 3$ steatosis between the NAFLD and ALD groups, as was reported previously. ${ }^{22}$ Also, NPV was similarly excellent in all patients and in each subgroup. Therefore, we not only presume that CAPs could detect more than $90 \%$ of patients with moderate or severe steatosis in US using these cutoff values, but also that patients with CAPs less than S2 or S3 cutoff values could be excluded from moderate or severe steatosis in US.

In our study, steatosis grade assessed by US and BMI were independently associated with CAP. Steatosis grade was the only common CAP-related independent parameter in both NAFLD and ALD groups and it was also the most important parameter with a prominent $\beta$ coefficient. In contrast, none of the other 
parameters including LSM and all of the laboratory parameters including ALT and total bilirubin were independently associated with CAP. These results indicated that CAP is remarkably related with hepatic steatosis and that unlike LSM, necroinflammatory activity dose not influence CAP values. All of these findings were consistent with other previous studies. ${ }^{16,21,23}$

Our study has a strength showing the relationship of CAP, a new promising tool, with US, the most commonly used tool, for assessing hepatic steatosis in over 180 fatty liver patients. Additionally, we compared this relationship, for the first time, in the NAFLD and ALD groups, which are major etiologies of hepatic steatosis. Assessing steatosis grade by US has a limitation by the subjective interpretation, thus CAP would help diagnosing fatty liver more accurately than US alone because CAP is expressed by quantified number and it is objective.

However, our study had several limitations. First, this study was retrospectively performed at a single center. Second, although US was examined by specialized radiologists, interobserver variability could exist. Third, we did not have biopsy samples which are regarded as the gold standard for staging as well as diagnosing hepatic steatosis. Lastly, it is quite difficult to define either NAFLD or ALD precisely because quantifying alcohol consumption is subjective and alcohol ingestion fluctuates over time. To overcome this problem, our study excluded patients whose diagnosis with either of these two diseases was ambiguous.

In conclusion, our study showed that CAP is relatively-well correlated with steatosis grades assessed by US regardless of hepatic steatosis etiology. From the results of our study, we can suggest that CAP is a promising modality not only for diagnosing but also quantifying hepatic steatosis compared with US, the most commonly used method, regardless of the causes of the disease. Our study also proposes cutoff values of CAP for each grade of steatosis with each etiology, but further large scale studies are needed to confirm the optimal cutoff values.

\section{CONFLICTS OF INTEREST}

None of the authors of this study has any financial interest or conflict with industries or parties.

\section{REFERENCES}

1. Levene AP, Goldin RD. The epidemiology, pathogenesis and histopathology of fatty liver disease. Histopathology 2012;61:141-152.

2. Lazo M, Clark JM. The epidemiology of nonalcoholic fatty liver disease: a global perspective. Semin Liver Dis 2008;28:339-350.

3. Bellentani S, Saccoccio G, Masutti F, et al. Prevalence of and risk factors for hepatic steatosis in Northern Italy. Ann Intern Med 2000;132:112-117.

4. Cerović I, Mladenović D, Ješić R, et al. Alcoholic liver disease/ nonalcoholic fatty liver disease index: distinguishing alcoholic from nonalcoholic fatty liver disease. Eur J Gastroenterol Hepatol 2013;25:899-904.

5. Myers RP, Pollett A, Kirsch R, et al. Controlled attenuation parameter (CAP): a noninvasive method for the detection of hepatic steatosis based on transient elastography. Liver Int 2012;32:902910.

6. Yeh MM, Brunt EM. Pathological features of fatty liver disease. Gastroenterology 2014;147:754-764.

7. Tuyama AC, Chang CY. Non-alcoholic fatty liver disease. J Diabetes 2012;4:266-280.

8. Starley BQ, Calcagno CJ, Harrison SA. Nonalcoholic fatty liver disease and hepatocellular carcinoma: a weighty connection. Hepatology 2010;51:1820-1832.

9. Sung KC, Wild SH, Kwag HJ, Byrne CD. Fatty liver, insulin resistance, and features of metabolic syndrome: relationships with coronary artery calcium in 10,153 people. Diabetes Care 2012;35: 2359-2364.

10. Rockey DC, Caldwell SH, Goodman ZD, Nelson RC, Smith AD; American Association for the Study of Liver Diseases. Liver biopsy. Hepatology 2009;49:1017-1044.

11. Grant A, Neuberger J. Guidelines on the use of liver biopsy in clinical practice. British Society of Gastroenterology. Gut 1999;45 Suppl 4:IV1-IV11.

12. Bohte AE, van Werven JR, Bipat S, Stoker J. The diagnostic accuracy of US, CT, MRI and 1H-MRS for the evaluation of hepatic steatosis compared with liver biopsy: a meta-analysis. Eur Radiol 2011;21:87-97.

13. Schwenzer NF, Springer F, Schraml C, Stefan N, Machann J, Schick F. Non-invasive assessment and quantification of liver steatosis by ultrasound, computed tomography and magnetic resonance. J Hepatol 2009;51:433-445.

14. Hamaguchi M, Kojima T, Itoh Y, et al. The severity of ultrasonographic findings in nonalcoholic fatty liver disease reflects the metabolic syndrome and visceral fat accumulation. Am J Gastroenterol 2007;102:2708-2715.

15. Dasarathy S, Dasarathy J, Khiyami A, Joseph R, Lopez R, McCullough AJ. Validity of real time ultrasound in the diagnosis of hepatic steatosis: a prospective study. J Hepatol 2009;51:10611067.

16. de Lédinghen V, Vergniol J, Capdepont M, et al. Controlled attenuation parameter (CAP) for the diagnosis of steatosis: a prospective study of 5323 examinations. J Hepatol 2014;60:1026-1031.

17. Sasso M, Beaugrand M, de Ledinghen V, et al. Controlled attenuation parameter (CAP): a novel $\mathrm{VCTE}^{\mathrm{TM}}$ guided ultrasonic attenuation measurement for the evaluation of hepatic steatosis. Preliminary study and validation in a cohort of patients with chronic liver disease from various causes. Ultrasound Med Biol 2010;36: 1825-1835.

18. Sasso M, Miette V, Sandrin L, Beaugrand M. The controlled attenuation parameter (CAP): a novel tool for the non-invasive evaluation of steatosis using Fibroscan. Clin Res Hepatol Gastroenterol 2012;36:13-20. 
19. Castera L, Forns X, Alberti A. Non-invasive evaluation of liver fibrosis using transient elastography. J Hepatol 2008;48:835-847.

20. de Lédinghen V, Vergniol J, Foucher J, Merrouche W, le Bail B. Non-invasive diagnosis of liver steatosis using controlled attenuation parameter (CAP) and transient elastography. Liver Int 2012; 32:911-918.

21. Chon YE, Jung KS, Kim SU, et al. Controlled attenuation parameter (CAP) for detection of hepatic steatosis in patients with chronic liver diseases: a prospective study of a native Korean population. Liver Int 2014;34:102-109.
22. Kumar M, Rastogi A, Singh T, et al. Controlled attenuation parameter for non-invasive assessment of hepatic steatosis: does etiology affect performance? J Gastroenterol Hepatol 2013;28:1194-1201.

23. Carvalhana S, Leitão J, Alves AC, Bourbon M, Cortez-Pinto H. How good is controlled attenuation parameter and fatty liver index for assessing liver steatosis in general population: correlation with ultrasound. Liver Int 2014;34:e111-e117.

24. Scaglioni F, Ciccia S, Marino M, Bedogni G, Bellentani S. ASH and NASH. Dig Dis 2011;29:202-210. 